\title{
Beziehungen zwischen Milchleistung und Fruchtbarkeit in einer Schwarzbuntpopulation nach intensiver Steigerung der Milch- leistung
}

Herrn Prof. Dr. agr. habil. Dr. h. c. mult. Georg Schönmuth zum 75. Geburtstag gewidmet

\begin{abstract}
Summary
Title of the paper: Relations between milk yield and fertility after strong increased milk yield

The purposes of the study were to estimate genetic parameters for milk production and fertility traits. The study included data on about 75,000 first and 40,000 second parity cows of German Friesian. The estimated coefficients of heritability for milk, fat and protein yield were in comparison to other previous estimates relatively high. In contrast to that, heritability of fat and protein content was very low. As expected, heritability of fertility also was very low, ranging from 0.009 to 0.127 , depending on trait. Genetic correlation between milk, fat and protein yield, and fertility traits were high and unfavourable and characterize an antagonistic relation. The estimated environmental correlation coefficients are negible. The genetic coefficients of regression between milk yield and fertility are low and vary between the pair of traits. Low genetic coefficients make the effect of antagonism less strong.
\end{abstract}

Key Words: Dairy cattle, milk yield, fertility, genetic correlation, environmental correlation, heritability

\section{Zusammenfassung}

An ca. 75000 Erst- und 40000 Zweitlaktationen von Kühen der Schwarzbuntpopulation in Mecklenburg-Vorpommern wurden die genetischen Parameter der Milchleistung und der Fruchtbarkeit geschätzt. Die geschätzten Heritabilitätskoeffizienten von Milch-, Milchfett- und Milcheiweißmenge sind in Vergleich zu Literaturwerten als hoch einzuschätzen, während für den Milchfett- und Milcheiweißgehalt unerwartet niedrige Schätzwerte gefunden wurden. Die Heritabilitätskoeffizienten der Fruchtbarkeitsmerkmale (Verzögerungszeit, Geburtsverlauf, Zwischentragezeit) erwiesen sich als sehr niedrig. Zwischen Milch-, Milchfett- und Milcheiweißmenge und den Fruchtbarkeitsmerkmalen wurden hohe, ungünstige genetische Korrelationen geschätzt, die auf einen Merkmalsantagonismus hinweisen. Die geschätzten Umweltkorrelationen erwiesen sich als unbedeutend. Die genetischen Regressionskoeffizienten von Milchleistung und Fruchtbarkeit sind niedrig und variieren zwischen den Merkmalspaaren. Sie sind z. T. sehr niedrig und entschärfen dadurch den Antagonismus.


lität

\section{Einleitung}

Die durchschnittliche Milchleistung der Milch- und Zweinutzungsrinder ist in Deutschland im letzten Jahrzehnt kontinuierlich gestiegen. Eine besonders intensive Steigerung ist in den neuen Bundesländern zu verzeichnen. Darunter nimmt Mecklenburg-Vorpommern mit einer Steigerung der Milchmengenleistung pro Kuh von 4290 $\mathrm{kg}$ im Jahre 1990 auf $7490 \mathrm{~kg}$ im Jahre 2000 einen vorderen Platz ein. Die Milchfettmenge erfuhr im gleichen Zeitraum eine Steigerung von $180 \mathrm{~kg}$ auf $320 \mathrm{~kg}$. Die 
große wirtschaftliche Bedeutung der Milchleistung für die Effektivität der Rinderproduktion lässt vermuten, dass auch zukünftig ein hoher Selektionsdruck zur Verbesserung der Milchleistung bestehen wird. Auch die Verminderung des Schadstoffeintrages je Produkteinheit in die Umwelt kann durch eine Leistungssteigerung weiter vorangetrieben werden.

In diesem Zusammenhang stellt sich die Frage, ob eine weitere Verbesserung der Milchleistung wegen des genetischen Antagonismus zu anderen Merkmalen noch möglich und ökonomisch sinnvoll ist. Damit gewinnt die in der Vergangenheit bereits oft diskutierte Frage der Beziehung von Milchleistung und Fruchtbarkeit beim Rind erneut aktuelle Bedeutung.

Die Beziehung zwischen der Milchleistung und der Fruchtbarkeit beim Rind hat viele Facetten. Eine gute Fruchtbarkeit ist wegen der regelmäßigen Kalbungen Voraussetzung für den Beginn einer neuen Laktation. Andererseits entsteht eine Konkurrenzsituation zwischen Fruchtbarkeit und Milchleistung, weil der Konzeptionszeitpunkt der Kühe in die Hochlaktation fällt. Die Analyse von Milchproduktionsbetrieben zeigte in der Vergangenheit allerdings immer wieder, dass in gut betreuten Herden neben einer hohen Milchleistung auch eine ausgezeichnete Fruchtbarkeit erreicht werden kann. Durch ein gutes Management lässt sich offenbar die Konkurrenzsituation um die zur Verfügung stehenden Nährstoffe im Tier weitgehend oder sogar vollkommen vermeiden. VEERKAMP et al. (2000) konnten experimentell nachweisen, dass die Energiebilanz der Kuh mit dem Beginn der Gelbkörperaktivitäten nach der Kalbung vorteilhaft $\left(\mathrm{r}_{\mathrm{g}}=-0,6\right)$ korreliert ist, während die Milchmenge sowie die Milchfett- und Milcheiweißmenge positiv $\left(\mathrm{r}_{\mathrm{g}}=0,51 ; 0,65\right.$ und 0,48$)$ und damit ungünstig mit dem Beginn der Gelbkörperaktivität nach der Kalbung verbunden sind. Erkenntnisse über die Wirkung des Leptins bei Milchkühen unterstützen die Bedeutung der Energiebilanz für die Beziehung zwischen Milchleistung und Fruchtbarkeit. Es ist u.a. für die Futteraufnahme, die Energiebilanz und die Fruchtbarkeit mit verantwortlich. Der Leptingenpolymorphismus RFLP1 ermöglicht eine Steigerung der Milchleistung (LIEFERS et al., 2002) ohne die Energiebilanz und damit die Fruchtbarkeit ungünstig zu beeinflussen.

Die Möglichkeit, die Konkurrenzsituation auch für eine zukünftige Steigerung der Milchleistung durch eine weitere Qualifizierung der Tierbetreuung zu meistern, wurde in der Vergangenheit häufig angezweifelt, wenn nicht gar ausgeschlossen und führte zu einem heftigen Meinungsstreit unter den Züchtern. Die vorliegende Untersuchung hat das Ziel, anhand der Milchrindpopulation des Zuchtverbandes Mecklenburg/Vorpommern die Beziehung zwischen Milchleistung und Fruchtbarkeit unter der gegenwärtigen Leistungshöhe zu analysieren und daraus Schlüsse für die Züchtung zu ziehen.

\section{2.} Material und Methoden

In die Untersuchungen wurden die Kühe der Geburtsjahrgänge 1995 bis 1997 des Rinderzuchtverbandes Mecklenburg/Vorpommern einbezogen. Die Beschränkung auf diese jungen Jahrgänge erfolgte, um ein genetisch einheitliches Tiermaterial hinsichtlich des Einsatzes von Schwarzbunt-Bullen mit einem hohen HF-Anteil in den neuen Bundesländern zu erhalten. Die Daten der Tiere wurden freundlicherweise vom VITVerden zur Verfügung gestellt. Eine detaillierte Beschreibung der Datenaufbereitung ist bei HENZE (2003) nachzulesen. Die Schätzung der genetischen und phänotypischen Parameter erfolgte nach REML mit analytischen Gradienten durch das Pro- 
gramm PEST (GROENEVELD et al., 1993) und VCE (NEUMAIER and GROENEVELD, 1998), wobei folgendes Modell verwendet worden ist:

$\begin{aligned} \mathrm{y}_{\mathrm{ijk}} & =\mu+\mathrm{B}_{\mathrm{i}}+\mathrm{JS}_{\mathrm{j}}+\mathrm{a}_{\mathrm{ijk}}+\mathrm{e}_{\mathrm{ijk}} \\ \mathrm{y}_{\mathrm{ijk}} & =\text { Beobachtungswert des Merkmals y } \\ \mu & =\text { Populationsmittel des Merkmals y } \\ \mathrm{B}_{\mathrm{i}}= & \text { fixer Effekt des Betriebes } \mathrm{i} \\ \mathrm{JS}_{\mathrm{j}} & =\text { fixer Effekt der Kalbesaison } \mathrm{j} \\ \mathrm{a}_{\mathrm{ijk}}= & \text { zufälliger Tiereffekt } \\ \mathrm{e}_{\mathrm{ijk}}= & \text { Zufallseffekt }\end{aligned}$

Neben den Milchleistungsmerkmalen der ersten und zweiten 305-Tage- Referenzlaktationen wurden als Merkmale aus dem Komplex der weiblichen Fruchtbarkeit der Geburtsverlauf (Bewertungsskala 1-5), die Verzögerungszeit (Zeitdifferenz zwischen der 1. und der erfolgreichen Besamung einer Kuh) und die Zwischenkalbezeit in die Analyse aufgenommen. Die Zwischenkalbezeit konnte wegen der zu geringen Anzahl von Tieren in der zweiten Laktation nur für die erste Laktation ausgewertet werden.

Ergebnisse

3.1 Mittelwerte, phänotypische und genetische Standardabweichungen und Heritabilitätskoeffizienten der Merkmale

In der Tabelle 1 sind die geschätzten genetischen und phänotypischen Parameter der Milchleistungs- und ausgewählter Fruchtbarkeitsmerkmale in der Erst- und Zweitlaktation dargestellt. Die durchschnittliche Milchmengenleistung der untersuchten Tiere in der ersten Laktation liegt noch knapp unter den oben angegebenen mittleren Leistungen aller Kühe des Jahres 2000. Die Leistungen in der zweiten Laktation liegen bereits über dem Leistungsdurchschnitt aller MLP-Kühe des Jahres 2000 und unterstreichen die Leistungsfähigkeit der jüngeren Kühe. Die Gehaltswerte an Milchfett und -eiweiß unterscheiden sich zwischen den Laktationen kaum.

Tabelle 1

Mittelwerte, genetische und phänotypische Standardabweichungen sowie Heritabilitätskoeffizienten von Milchleistungs- und Fruchtbarkeitsmerkmalen in der 1. und 2. Laktation (Means, genetic and phenotypic standard deviations and heritability of milk performance and fertility traits in the $1^{\text {th }}$ and $2^{\text {nd }}$ lactation)

\begin{tabular}{|c|c|c|c|c|c|c|c|c|c|}
\hline & $\begin{array}{l}\text { Lak- } \\
\text { tation }\end{array}$ & $\begin{array}{l}\text { Milch- } \\
\text { menge } \\
\text { kg }\end{array}$ & $\begin{array}{c}\text { Fett- } \\
\text { menge } \\
\text { kg }\end{array}$ & $\begin{array}{c}\text { Eiweiß- } \\
\text { menge } \\
\text { kg }\end{array}$ & $\begin{array}{c}\text { Fett- } \\
\text { gehalt } \\
\%\end{array}$ & $\begin{array}{c}\text { Eiweiß- } \\
\text { gehalt } \\
\%\end{array}$ & $\begin{array}{c}\text { Verzöge- } \\
\text { rungszeit } \\
\text { Tage }\end{array}$ & $\begin{array}{c}\text { Geburts- } \\
\text { verlauf }\end{array}$ & $\begin{array}{l}\text { ZKZ } \\
\text { Tage }\end{array}$ \\
\hline \multirow[t]{2}{*}{$\mathrm{n}$} & 1 . & 75419 & 75415 & 75397 & 75419 & 75419 & 96644 & 128254 & \multirow[t]{2}{*}{56182} \\
\hline & 2. & 40232 & 40232 & 40232 & 40231 & 40232 & 58582 & 72075 & \\
\hline \multirow[b]{2}{*}{$\bar{x}$} & 1. & 7079,0 & 294,0 & 244,4 & 4,199 & 3,467 & 33 & 1,250 & \multirow[t]{2}{*}{394,4} \\
\hline & 2. & 8408,0 & 349,7 & 290,4 & 4,196 & 3,473 & 35 & 1,100 & \\
\hline \multirow[t]{2}{*}{$\mathrm{S}_{\mathrm{g}}$} & 1. & 933,9 & 34,7 & 27,8 & 0,340 & 0,120 & 4,603 & 0,174 & \multirow[t]{2}{*}{12,6} \\
\hline & 2. & 1111,2 & 37,6 & 32,7 & 0,349 & 0,126 & 6,761 & 0,068 & \\
\hline \multirow[t]{2}{*}{$\mathrm{s}_{\mathrm{p}}$} & 1. & 1462,0 & 54,2 & 47,0 & 0,500 & 0,210 & 48,5 & 0,489 & \multirow[t]{2}{*}{60,5} \\
\hline & 2. & 1768,0 & 67,1 & 55,6 & 0,538 & 0,224 & 49,0 & 0,332 & \\
\hline \multirow[b]{2}{*}{$\hat{\mathrm{h}}^{2}$} & 1.* & 0,408 & 0,409 & 0,349 & 0,474 & 0,353 & 0,009 & 0,127 & \multirow[t]{2}{*}{0,043} \\
\hline & 2.* & 0,395 & 0,314 & 0,346 & 0,420 & 0,317 & 0,019 & 0,042 & \\
\hline
\end{tabular}

Die Schätzwerte für die Heritabilität der Milchmengeneigenschaften fallen relativ hoch aus, während für den Milchfett- und Milcheiweißgehalt entgegen den Erwartun- 
gen recht niedrige Schätzwerte gefunden worden sind. In der zweiten Laktation sind die Schätzwerte für die Heritabilitätskoeffizienten aller Milchleistungsmerkmale niedriger als in der ersten Laktation. Für die Heritabilität der Fruchtbarkeitsmerkmale wurden die allgemein zu erwartenden niedrigen Schätzwerte bestätigt. Die Heritabilität des Geburtsverlaufs der 1. Kalbung erreicht einen für Fruchtbarkeitsmerkmale hohen Schätzwert, der sich jedoch in der 2. Kalbung nicht bestätigt. Aufgrund der hohen phänotypischen Varianz der Fruchtbarkeitsmerkmale ist trotz ihrer geringen Heritabilität, mit Ausnahme des Geburtsverlaufs, noch eine beachtliche genetische Varianz in der Population vorhanden, die für eine züchterische Merkmalsverbesserung nutzbar ist.

\subsection{Phänotypische Beziehungen}

Die phänotypischen Beziehungen der Milchleistungsmerkmale zeichnen sich durch eine enge Korrelation der Mengenmerkmale untereinander aus, während der Milchfettund Milcheiweißgehalt signifikant negativ mit der Milchmenge korreliert sind und die Korrelationskoeffizienten im oberen mittleren Bereich eine mittelstraffe ungünstige Beziehung darstellen (Tab. 2). Der Milchfettgehalt ist mit der Milchfettmenge zwar niedrig, aber in beiden Laktationen positiv korreliert, dagegen ist die Korrelation zwischen Milcheiweißgehalt und Milcheiweißmenge in den beiden Laktationen uneinheitlich. Die Korrelationskoeffizienten zwischen Fruchtbarkeits- und Milchmengenmerkmalen sind positiv und bringen damit eine ungünstige Verbindung zum Ausdruck. Sie sind aber alle kleiner als 0,07 und nur wegen des großen Stichprobenumfangs signifikant von Null verschieden. Die phänotypischen Korrelationen zwischen den Fruchtbarkeitsmerkmalen und den Milchinhaltsstoffen sind noch niedriger und mit wechselnden Vorzeichen zwischen den Laktationen.

Tabelle 2

Phänotypische Korrelationskoeffizienten zwischen Milchleistungs- und Fruchtbarkeitsmerkmalen in der 1. und 2. Laktation (Phenotypic correlation coefficients between milk performance and fertility traits in the $1^{\text {th }}$ and $2^{\text {nd }}$ lactation)

\begin{tabular}{|c|c|c|c|c|c|c|c|c|}
\hline & $\begin{array}{l}\text { Lak- } \\
\text { tation }\end{array}$ & $\begin{array}{c}\text { Fett- } \\
\text { menge } \\
\text { kg }\end{array}$ & $\begin{array}{c}\text { Eiweiß- } \\
\text { menge } \\
\mathrm{kg}\end{array}$ & $\begin{array}{c}\text { Fett- } \\
\text { gehalt } \\
\% \\
\end{array}$ & $\begin{array}{c}\text { Eiweiß- } \\
\text { gehalt } \\
\%\end{array}$ & $\begin{array}{c}\text { Verzöge- } \\
\text { rungszeit } \\
\text { Tage }\end{array}$ & $\begin{array}{c}\text { Geburts- } \\
\text { verlauf }\end{array}$ & $\begin{array}{l}\text { ZKZ } \\
\text { Tage }\end{array}$ \\
\hline \multirow{2}{*}{$\begin{array}{l}\text { Milch- } \\
\text { menge }\end{array}$} & 1. & $0,807^{*}$ & $0,955^{*}$ & $-0,448 *$ & $-0,347^{*}$ & $0,050^{*}$ & 0,006 & $0,053^{*}$ \\
\hline & 2. & $0,786^{*}$ & $0,952 *$ & $-0,433^{*}$ & $-0,415^{*}$ & $0,059 *$ & 0,004 & \\
\hline \multirow{2}{*}{$\begin{array}{l}\text { Fett- } \\
\text { menge }\end{array}$} & 1. & & $0,865^{*}$ & $0,149 *$ & $0,008 *$ & $0,053^{*}$ & $0,011^{*}$ & $0,045^{*}$ \\
\hline & 2. & & $0,851 *$ & $0,198^{*}$ & $-0,034^{*}$ & $0,059 *$ & 0,020 & \\
\hline \multirow{2}{*}{$\begin{array}{l}\text { Eiweiß- } \\
\text { menge }\end{array}$} & 1. & & & $-0,287^{*}$ & $-0,062 *$ & $0,055^{*}$ & 0,006 & $0,043 *$ \\
\hline & 2. & & & $-0,266^{*}$ & $0,585^{*}$ & $0,065^{*}$ & 0,007 & \\
\hline \multirow{2}{*}{$\begin{array}{l}\text { Fett- } \\
\text { gehalt }\end{array}$} & 1. & & & & $0,621 *$ & $-0,006^{*}$ & $0,006^{*}$ & $-0,025^{*}$ \\
\hline & 2. & & & & $0,637 *$ & $-0,009$ & $-0,004$ & \\
\hline \multirow{2}{*}{$\begin{array}{l}\text { Eiweiß- } \\
\text { gehalt }\end{array}$} & 1. & & & & & 0,003 & $-0,002$ & $-0,047^{*}$ \\
\hline & 2. & & & & & $-0,002$ & 0,005 & \\
\hline \multirow{2}{*}{$\begin{array}{l}\text { Verzöge- } \\
\text { rungszeit }\end{array}$} & 1. & & & & & & $0,024^{*}$ & $0,740 *$ \\
\hline & 2. & & & & & & $0,028^{*}$ & \\
\hline $\begin{array}{l}\text { Geburts- } \\
\text { verlauf }\end{array}$ & $\begin{array}{l}1 . \\
2 .\end{array}$ & & & & & & & $0,031 *$ \\
\hline
\end{tabular}

Die phänotypischen Regressionskoeffizienten für die Milchmenge und die Verzögerungszeit sowie für alle Milchmengeneigenschaften und den Geburtsverlauf in 
der ersten Laktation sind kleiner als ein Tausendstel (Tab. 3). Eine Steigerung der Milchmengenleistung um 1000kg würde demnach in der Population die Verzögerungszeit um weniger als einen Tag verlängern, die Zwischenkalbezeit dagegen um 2 Tage. Der Regressionskoeffizient des Fettgehaltes auf die Verzögerungszeit ist zwar negativ und damit günstig im Sinne des Zuchtzieles, allerdings reduziert sich die Verzögerungszeit bei einer Steigerung des Fettgehaltes um $1 \%$ in der ersten bzw. zweiten Laktation durchschnittlich um nur 0,6 bis 0,8 Tage und bleibt damit marginal im Vergleich zur Veränderung des Milchfettgehaltes. Die Regressionskoeffizienten des Eiweißgehaltes in der ersten und zweiten Laktation haben unterschiedliche Vorzeichen und sind beide nicht signifikant von Null verschieden. Innerhalb der Fruchtbarkeitsmerkmale wird die Abhängigkeit der Zwischenkalbezeit von der Verzögerungszeit durch den Korrelationskoeffizienten von $\mathrm{r}=0,74$ unterstrichen (Tab.2). Der entsprechende Regressionskoeffizient von 0,923 zeigt, dass die Verlängerung der Verzögerungszeit fast direkt an die Zwischenkalbezeit weitergegeben wird (Tab.3).

Tabelle 3

Phänotypische Regressionskoeffizienten zwischen Milchleistungs- und Fruchtbarkeitsmerkmalen der 1. und 2. Laktation (Phenotypic regression coefficients between milk performance and fertility traits in the $1^{\text {th }}$ and $2^{\text {nd }}$ lactation)

\begin{tabular}{|c|c|c|c|c|c|c|c|c|}
\hline & $\begin{array}{l}\text { Lak- } \\
\text { tation }\end{array}$ & $\begin{array}{l}\text { Fett- } \\
\text { menge } \\
\text { kg }\end{array}$ & $\begin{array}{l}\text { Eiweiß- } \\
\text { menge } \\
\text { kg }\end{array}$ & $\begin{array}{c}\text { Fett- } \\
\text { gehalt } \\
\%\end{array}$ & $\begin{array}{c}\text { Eiweiß- } \\
\text { gehalt } \\
\%\end{array}$ & $\begin{array}{l}\text { Verzöge- } \\
\text { rungszeit } \\
\text { Tage }\end{array}$ & $\begin{array}{l}\text { Geburts- } \\
\text { verlauf }\end{array}$ & $\begin{array}{l}\text { ZKZ } \\
\text { Tage }\end{array}$ \\
\hline \multirow{2}{*}{$\begin{array}{l}\text { Milch- } \\
\text { menge }\end{array}$} & 1. & 0,030 & 0,031 & 0,000 & 0,000 & 0,000 & 0,000 & 0,002 \\
\hline & 2. & 0,030 & 0,030 & 0,000 & 0,000 & 0,002 & 0,000 & \\
\hline \multirow{2}{*}{$\begin{array}{l}\text { Fett- } \\
\text { menge }\end{array}$} & 1. & & 0,750 & 0,001 & 0,000 & 0,047 & 0,000 & 0,050 \\
\hline & 2. & & 0,706 & 0,001 & 0,000 & 0,043 & 0,000 & \\
\hline \multirow{2}{*}{$\begin{array}{c}\text { Eiweiß- } \\
\text { menge }\end{array}$} & 1. & & & $-0,003$ & 0,000 & 0,057 & 0,000 & 0,055 \\
\hline & 2. & & & $-0,002$ & 0,002 & 0,057 & 0,000 & \\
\hline \multirow{2}{*}{$\begin{array}{l}\text { Fett- } \\
\text { gehalt }\end{array}$} & 1. & & & & 0,261 & $-0,582$ & 0,006 & $-3,026$ \\
\hline & 2. & & & & 0,259 & $-0,818$ & $-0,002$ & \\
\hline \multirow{2}{*}{$\begin{array}{c}\text { Eiweiß- } \\
\text { gehalt }\end{array}$} & 1. & & & & & 0,693 & $-0,005$ & $-13,5$ \\
\hline & 2. & & & & & $-0,446$ & 0,007 & \\
\hline \multirow{2}{*}{$\begin{array}{l}\text { Verzöge- } \\
\text { rungszeit }\end{array}$} & 1. & & & & & & 0,000 & 0,923 \\
\hline & 2. & & & & & & 0,000 & \\
\hline $\begin{array}{c}\text { Geburts- } \\
\text { verlauf }\end{array}$ & $\begin{array}{l}1 . \\
2 .\end{array}$ & & & & & & & 3,806 \\
\hline
\end{tabular}

\section{3} Genetische Beziehungen

Die genetischen Korrelations- und Regressionskoeffizienten (Tab. 4 u. 5) widerspiegeln den Grad genetischer Abhängigkeit der Merkmale und damit die züchterisch wichtigere Komponente der Merkmalsbeziehungen. Innerhalb der Milchleistungsmerkmale ist zwischen der Milchmenge und den Gehaltswerten in beiden Laktationen eine hohe negative Korrelation vorhanden, die Ausdruck eines Merkmalsantagonismus zwischen Milchleistung und Fruchtbarkeit ist. Die Milchmengeneigenschaften haben sowohl mit der Verzögerungszeit als auch mit der Zwischenkalbezeit mittlere bis hohe positive genetische Korrelationen, die ebenfalls einen Merkmalsantagonismus widerspiegeln. Die genetischen Korrelationen der Gehaltswerte (Milchfett und Milcheiweiß) zu den Fruchtbarkeitsmerkmalen (Verzögerungszeit und Zwischenkalbezeit) sind negativ und daher als günstig anzusehen, da sich ein höherer Konzentrationsgrad der Milch positiv auf die Fruchtbarkeit auswirkt. 
Tabelle 4

Genetische Korrelationskoeffizienten zwischen Milchleistungs- und Fruchtbarkeitsmerkmalen in der 1 . und 2. Laktation (Genetic correlation coefficients between milk performance and fertility traits in the $1^{\text {th }}$ and $2^{\text {nd }}$ lactation)

\begin{tabular}{|c|c|c|c|c|c|c|c|c|}
\hline & $\begin{array}{c}\text { Lak- } \\
\text { tation }\end{array}$ & $\begin{array}{c}\text { Fett- } \\
\text { menge } \\
\text { kg }\end{array}$ & $\begin{array}{c}\text { Eiweiß- } \\
\text { menge } \\
\mathrm{kg}\end{array}$ & $\begin{array}{c}\text { Fett- } \\
\text { gehalt } \\
\%\end{array}$ & $\begin{array}{c}\text { Eiweiß- } \\
\text { gehalt } \\
\%\end{array}$ & $\begin{array}{c}\text { Verzöge- } \\
\text { rungszeit } \\
\text { Tage }\end{array}$ & $\begin{array}{c}\text { Geburts- } \\
\text { verlauf }\end{array}$ & $\begin{array}{l}\text { ZKZ } \\
\text { Tage }\end{array}$ \\
\hline \multirow{2}{*}{$\begin{array}{l}\text { Milch- } \\
\text { menge }\end{array}$} & 1. & $0,579^{*}$ & $0,907^{*}$ & $-0,614^{*}$ & $-0,600^{*}$ & 0,593 & $0,028^{*}$ & $0,635^{*}$ \\
\hline & 2. & $0,530 *$ & $0,910 *$ & $-0,687 *$ & $-0,580^{*}$ & 0,504 & 0,085 & \\
\hline \multirow{2}{*}{$\begin{array}{l}\text { Fett- } \\
\text { menge }\end{array}$} & 1. & & $0,760^{*}$ & $0,269 *$ & $0,017^{*}$ & 0,433 & $0,096^{*}$ & $0,510^{*}$ \\
\hline & 2. & & $0,710^{*}$ & $0,296 *$ & $0,113^{*}$ & $0,280 *$ & $0,152 *$ & \\
\hline \multirow{2}{*}{$\begin{array}{l}\text { Eiweiß- } \\
\text { menge }\end{array}$} & 1. & & & $-0,365^{*}$ & $-0,254^{*}$ & 0,550 & $0,016^{*}$ & $0,606^{*}$ \\
\hline & 2. & & & $-0,411 *$ & $-0,237 *$ & $0,465 *$ & $0,133^{*}$ & \\
\hline \multirow{2}{*}{$\begin{array}{l}\text { Fett- } \\
\text { gehalt }\end{array}$} & 1. & & & & $0,772 *$ & $-0,287$ & $0,009^{*}$ & $-0,310$ \\
\hline & 2. & & & & $0,813 *$ & $-0,313$ & $0,012 *$ & \\
\hline \multirow{2}{*}{$\begin{array}{l}\text { Eiweiß- } \\
\text { gehalt }\end{array}$} & 1. & & & & & $-0,230$ & $-0,031^{*}$ & $-0,364^{*}$ \\
\hline & 2. & & & & & $-0,226^{*}$ & $0,076^{*}$ & \\
\hline \multirow{2}{*}{$\begin{array}{l}\text { Verzöge- } \\
\text { rungszeit }\end{array}$} & 1. & & & & & & 0,405 & $0,856^{*}$ \\
\hline & 2. & & & & & & 0,277 & \\
\hline $\begin{array}{l}\text { Geburts- } \\
\text { verlauf }\end{array}$ & $\begin{array}{l}1 . \\
2 .\end{array}$ & & & & & & & $0,233^{*}$ \\
\hline
\end{tabular}

Tabelle 5

Genetische Regressionskoeffizienten zwischen Milchleistungs- und Fruchtbarkeitsmerkmalen der 1. und 2. Laktation (Genetic regression coefficients between milk performance and fertility traits in the $1^{\text {th }}$ and $2^{\text {nd }}$ lactation)

\begin{tabular}{|c|c|c|c|c|c|c|c|c|}
\hline & $\begin{array}{c}\text { Lak- } \\
\text { tation }\end{array}$ & $\begin{array}{c}\text { Fett- } \\
\text { menge } \\
\text { kg }\end{array}$ & $\begin{array}{c}\text { Eiweiß- } \\
\text { menge } \\
\text { kg }\end{array}$ & $\begin{array}{c}\text { Fett- } \\
\text { gehalt } \\
\% \\
\end{array}$ & $\begin{array}{c}\text { Eiweiß- } \\
\text { gehalt } \\
\%\end{array}$ & $\begin{array}{l}\text { Verzöge- } \\
\text { rungszeit } \\
\text { Tage }\end{array}$ & $\begin{array}{l}\text { Geburts- } \\
\text { verlauf }\end{array}$ & $\begin{array}{l}\text { ZKZ } \\
\text { Tage }\end{array}$ \\
\hline \multirow{2}{*}{$\begin{array}{l}\text { Milch- } \\
\text { menge }\end{array}$} & 1. & 0,021 & 0,027 & 0,000 & 0,000 & 0,003 & 0,000 & 0,009 \\
\hline & 2. & 0,018 & 0,027 & 0,000 & 0,000 & 0,003 & 0,000 & \\
\hline \multirow{2}{*}{$\begin{array}{l}\text { Fett- } \\
\text { menge }\end{array}$} & 1. & & 0,608 & 0,003 & 0,000 & 0,057 & 0,001 & 0,185 \\
\hline & 2. & & 0,618 & 0,003 & 0,000 & 0,050 & 0,000 & \\
\hline \multirow{2}{*}{$\begin{array}{l}\text { Eiweiß- } \\
\text { menge }\end{array}$} & 1. & & & $-0,004$ & $-0,001$ & 0,091 & 0,000 & 0,274 \\
\hline & 2. & & & $-0,004$ & 0,000 & 0,096 & 0,000 & \\
\hline \multirow{2}{*}{$\begin{array}{c}\text { Fett- } \\
\text { gehalt }\end{array}$} & 1. & & & & 0,272 & $-3,883$ & $-0,005$ & $-11,4$ \\
\hline & 2. & & & & 0,278 & $-6,045$ & 0,002 & \\
\hline \multirow{2}{*}{$\begin{array}{c}\text { Eiweiß- } \\
\text { gehalt }\end{array}$} & 1. & & & & & $-8,817$ & $-0,047$ & $-38,1$ \\
\hline & 2. & & & & & $-12,7$ & 0,044 & \\
\hline \multirow{2}{*}{$\begin{array}{l}\text { Verzöge- } \\
\text { rungszeit }\end{array}$} & 1. & & & & & & 0,016 & 2,335 \\
\hline & 2. & & & & & & 0,003 & \\
\hline $\begin{array}{l}\text { Geburts- } \\
\text { verlauf }\end{array}$ & $\begin{array}{l}1 . \\
2 .\end{array}$ & & & & & & & 16,2 \\
\hline
\end{tabular}

Wie stark sich die weitere züchterische Steigerung der Milchleistung auf die Fruchtbarkeit auswirkt, widerspiegelt die Höhe der genetischen Regressionskoeffizienten (Tab. 5). In der ersten Laktation verlängert eine Steigerung der Milchleistung um 1000kg Milch die Verzögerungszeit im Mittel um 3 und die Zwischenkalbezeit um 9 Tage. Eine züchterische Verbesserung der Milchfettmenge um 100kg würde eine Verlängerung der Verzögerungszeit um 5,7 Tage verursachen, während die Zwischenkalbezeit um 18,5 Tage ansteigen würde. Eine ebensolche Erhöhung der Milcheiweißmenge würde die Verzögerungszeit um 9,1 und die Zwischenkalbezeit um 27,4 Tage verlängern. 
Beim Fett- und Eiweißgehalt ist, wie auf Grund der genetischen Korrelation zu erwarten, durch eine Erhöhung ein positiver Effekt auf die Verzögerungs- und Zwischenkalbezeit zu erreichen. Die Höhe des Regressionskoeffizienten relativiert jedoch den zu erzielenden Effekt. Eine Erhöhung des Milchfettgehaltes um 1\% führt zu einer Verkürzung der Verzögerungszeit um nahezu 4 Tage und der Zwischenkalbezeit um 11,4 Tage. Eine gleiche Steigerung des Eiweißgehaltes würde sogar zu einer Verkürzung um 8,8 bzw. 38,1 bei der Verzögerungs- bzw. Zwischenkalbezeit führen. In der zweiten Laktation sind, möglicherweise bedingt durch die höheren Leistungen, die Auswirkungen auf die Verzögerungszeit deutlich stärker. Der Einfluss der Milchleistungsmerkmale auf den Geburtsverlauf ist sowohl qualitativ als auch quantitativ schwach und nicht eindeutig.

\subsection{Umweltbedingte Abhängigkeiten}

Anhand der geschätzten Umweltkorrelationen (Tab. 6) kann eine Aussage über die umweltbedingte Abhängigkeit der Merkmale getroffen werden. Zwischen allen Fruchtbarkeits- und Milchleistungsmerkmalen bestehen außerordentlich geringe, durch die Umwelt bedingte Abhängigkeiten. Die meisten geschätzten Korrelationen liegen weit unter 0,05. Innerhalb der Fruchtbarkeitsmerkmale gibt es aber deutliche Beziehungen aufgrund gemeinsamer Umwelteffekte, so z.B. zwischen Verzögerungszeit und Zwischenkalbezeit mit einer Umweltkorrelation von 0,743. Auch unter den Milchleistungsmerkmalen sind umweltbedingte Abhängigkeiten nachgewiesen. Die Mengeneigenschaften sind in beiden Laktationen eng korreliert $(r>0,9)$. Fett- und Eiweißgehalt sind durch eine Umweltkorrelation von $r_{u}=0,661$ bei der ersten und mit $r_{u}=0,582$ in der zweiten Laktation verbunden.

\section{Tabelle 6}

Umweltkorrelationskoeffizienten zwischen Milchleistungs- und Fruchtbarkeitsmerkmalen der 1. und 2. Laktation (Environmental correlation coefficients between milk performance and fertility traits in the $1^{\text {th }}$ and $2^{\text {nd }}$ lactation)

\begin{tabular}{|c|c|c|c|c|c|c|c|c|}
\hline & $\begin{array}{c}\text { Lak- } \\
\text { tation }\end{array}$ & $\begin{array}{c}\text { Fett- } \\
\text { menge } \\
\text { kg }\end{array}$ & $\begin{array}{c}\text { Eiweiß- } \\
\text { menge } \\
\text { kg }\end{array}$ & $\begin{array}{c}\text { Fett- } \\
\text { gehalt } \\
\% \\
\end{array}$ & $\begin{array}{c}\text { Eiweiß- } \\
\text { gehalt } \\
\% \\
\end{array}$ & $\begin{array}{l}\text { Verzöge- } \\
\text { rungszeit } \\
\text { Tage }\end{array}$ & $\begin{array}{c}\text { Geburts- } \\
\text { verlauf }\end{array}$ & $\begin{array}{l}\text { ZKZ } \\
\text { Tage }\end{array}$ \\
\hline \multirow{2}{*}{$\begin{array}{l}\text { Milch- } \\
\text { menge }\end{array}$} & 1. & 0,964 & 0,987 & $-0,327$ & $-0,247$ & 0,018 & $-0,001$ & $-0,041$ \\
\hline & 2. & 0,930 & 0,979 & $-0,258$ & $-0,353$ & 0,020 & $-0,009$ & \\
\hline \multirow{2}{*}{$\begin{array}{l}\text { Fett- } \\
\text { menge }\end{array}$} & 1. & & 0,932 & 0,058 & 0,003 & 0,035 & $-0,016$ & $-0,030$ \\
\hline & 2. & & 0,921 & 0,143 & $-0,105$ & 0,046 & 0,002 & \\
\hline \multirow{2}{*}{$\begin{array}{l}\text { Eiweiß- } \\
\text { menge }\end{array}$} & 1. & & & $-0,241$ & 0,044 & 0,030 & 0,003 & $-0,040$ \\
\hline & 2. & & & 0,252 & 0,123 & $-0,047$ & 0,020 & \\
\hline \multirow{2}{*}{$\begin{array}{l}\text { Fett- } \\
\text { gehalt }\end{array}$} & 1. & & & & 0,661 & 0,017 & 0,006 & 0,027 \\
\hline & 2. & & & & 0,582 & 0,025 & $-0,007$ & \\
\hline \multirow{2}{*}{$\begin{array}{c}\text { Eiweiß- } \\
\text { gehalt }\end{array}$} & 1. & & & & & 0,023 & 0,007 & $-0,006$ \\
\hline & 2. & & & & & 0,019 & $-0,005$ & \\
\hline \multirow{2}{*}{$\begin{array}{l}\text { Verzöge- } \\
\text { rungszeit }\end{array}$} & 1. & & & & & & 0,011 & 0,743 \\
\hline & 2. & & & & & & 0,020 & \\
\hline $\begin{array}{l}\text { Geburts- } \\
\text { verlauf }\end{array}$ & $\begin{array}{l}1 . \\
2 .\end{array}$ & & & & & & & 0,015 \\
\hline
\end{tabular}

4. Diskussion

Die Fruchtbarkeit beim Milchrind wird vielfach als kritisch angesehen, weil die Beziehungen zur Milchleistung ungünstig sind, und wegen der aus wirtschaftlichen Gründen 
erhöhten Milchleistung ist in einigen Populationen bereits ein negativer genetischer Trend in den Fruchtbarkeitsmerkmalen beobachtet wurde (ROYAL et al., 2002). In den neuen Bundesländern erfolgte von 1990 bis 2000 ein besonders starker Anstieg der Milchleistung, der sowohl auf genetisch-züchterische Ursachen (Einsatz von Bullen mit hohen HF-Anteil) als auch auf Umwelteffekte (Verbesserung der Futterqualität) zurückzuführen ist (SCHÖNMUTH, 1997). Daraus ergibt sich die Frage, ob sich der Antagonismus zwischen Milchleistung und Fruchtbarkeit möglicherweise verstärkt hat und welche züchterischen Möglichkeiten zur Verbesserung der Milchleistung und evtl. auch der Fruchtbarkeit in der Population vorhanden sind. Die in den eigenen Untersuchungen geschätzten Heritabilitätskoeffizienten der Milchleistungsmerkmale liegen für die Milch-, Milchfett- und Milcheiweißmenge höher als in vergleichbaren Untersuchungen (DEMATAWEWA and BERGER, 1998; EVANS et al., 2002; CASTILLO-JUAREZ et al., 2002) anderer Populationen. Eine starke Erhöhung der Milchleistung in den vergangenen Jahren hat demnach nicht zu einer Einschränkung der genetischen Varianz geführt, die den künftigen Zuchtfortschritt schmälern würde. Eine Plateaubildung wäre nach der Anpaarung von unverwandten Bullen mit hohem HF-Anteil an die heimische Population auch nicht zu erwarten. Die niedrigen Heritabilitätskoeffizienten des Milchfett- und Milcheiweißgehaltes sind allerdings nicht plausibel zu erklären. Die niedrigen Heritabilitätskoeffizienten für die Merkmale der Fruchtbarkeit stimmen mit den Schätzwerten der neueren Literatur überein (DEMATAWEWA and BERGER, 1998; ROTH et al., 1999; VEERKAMP et al., 2000; WASSMUTH et al., 2000). Dass bei einigen Fruchtbarkeitsmerkmalen trotz der niedrigen Heritabilität ein genetischer Variationskoeffizient vorhanden ist, der mit dem der Milchleistungsmerkmale durchaus vergleichbar ist, betonen ROYAL et al. (2002). Das wird in den eigenen Untersuchungen für die Merkmale Verzögerungszeit und Geburtsverlauf bestätigt und unterstreicht die potentiellen Möglichkeiten einer züchterischen Verbesserung von Fruchtbarkeitsmerkmalen.

Die genetischen Beziehungen der Milchmenge, Milchfett- und Milcheiweißmenge zur Verzögerungszeit und Zwischenkalbezeit sind durch eine positive und damit ungünstige genetische Korrelation in einer Höhe von 0,433 bis 0,635 charakterisiert. Sie unterstreichen damit den Antagonismus zwischen diesen Merkmalskomplexen und stimmen dahingehend mit den Ergebnissen von DEMATAWEWA and BERGER (1998) und EVENS et al. (2002), überein, während die genetischen Korrelationen nach ROCKSTRÖM et al. (2001) im Bereich von 0,2 bis 0,4 liegen. Der Geburtsverlauf scheint genetisch von der Milchleistung weitgehend unabhängig zu sein.

Die indirekten genetischen Veränderungen der Fruchtbarkeitsmerkmale infolge einer weiteren Steigerung der Milchleistung, widergespiegelt durch die genetischen Regressionskoeffizienten, relativiert jedoch den Antagonismus der Milchleistung zu den einzelnen Fruchtbarkeitsmerkmalen erheblich (SCHÖNMUTH, 1997). Die Auswirkungen auf die Verzögerungszeit sind, bedingt durch die z.T. sehr niedrigen genetischen Regressionskoeffizienten, besonders bei der Erhöhung der Milchmenge, sehr moderat. Ähnlich niedrige genetische Regressionskoeffizienten zwischen Fruchtbarkeits- und Milchleistungsmerkmalen fanden ROYAL et al. ( 2002). Die Verlängerung der Zwischenkalbezeit ist dagegen beachtlich und betont den Merkmalsantagonismus. Dagegen wirkt sich eine Erhöhung des Milchfett- und des Milcheiweißgehaltes günstig auf die Fruchtbarkeitsmerkmale aus, wie an den negativen Vorzeichen der genetischen Korrelationskoeffizienten zu erkennen ist, die in beiden Laktationen signifikant von 
Null verschieden sind. Die Höhe der genetischen Regressionskoeffizienten zeigt jedoch, dass die Wirkungen einer konzentrierteren Milch auf die Fruchtbarkeitsleistung nur marginal bleibt.

Die niedrigen Umweltkorrelationen zwischen Milch- und Fruchtbarkeitsmerkmalen könnten generell gegen die These sprechen, dass beide Merkmalskomplexe konkurrieren. Ebenso niedrige Werte wurden von DEMATAWEWA and BERGER (1998) gefunden. Etwas höher, aber ebenfalls noch im niedrigen Bereich liegen die Schätzwerte von ROYAL et al. ( 2002). Die geringen Umweltkorrelationen könnten aber auch Ausdruck einer durch ausreichend angebotene Nährstoffe weitgehend befriedigten Konkurrenzsituation sein, obwohl die „Konkurrenzthese“ im Allgemeinen gilt. Die von VEERKAMP et al. (2000) nachgewiesene negative, aber günstige Korrelation zwischen der Energiebilanz und der Fruchtbarkeit stützen die Konkurrenzhypothese. Die Möglichkeit, eine Konkurrenzsituation auch genetisch über den Leptin- Polymorphismus zu vermeiden, spricht dafür, dass in der Zuchtpraxis beide Möglichkeiten, züchterische und Umweltverbesserung, genutzt werden sollten.

\section{Literatur}

CASTILLO-JUAREZ, H.; OLTENACU, P.A.; CIENFUEGOS-RIVAS, E.G:

Genetic and phenotypic relationships among milk production and composition traits in primiparous Holstein cows in two different herd invironments. Livestock Prod. Sci. 78 (2002), 223-231

DEMATAWEWA, C.M.B.; BERGER, P.J.:

Genetic and Phenotypic Parameters for 305-Day Yield, Fertility, and Survival in Holsteins. J. Dairy Sci. 81 (1998), 2700-2709

EVANS, R.D.; BUCKLEY, F.; DILLON, P.; VEERKAMP, R.F.:

Genetic parameters for production and fertility in spring-calving Irish dairy cattle. Irish Journal of Agricultural and Food Research 41 (2002), 43-54

GROENEVELD, E.; KOVAC, M.; WANG, T.:

PEST-Multivariate Predicion and Estimation. Department of Animal Sciences, University of Illinois, 1993

HENZE, C.:

Bedeutung der Milchleistungsmerkmale bei Wegfall der Milchquotenregelung in der Europäischen Union. Humboldt-Universität zu Berlin, Diss., 2003

LIEFERS, S.C.; TE PAS, M.F.W.; VEERKAMP, R.F.; VAN DER LENDE, T.:

Associations between Leptin Gene Polymorhismus and Production, Live Weight, Energy Balance, Feed Intake, and Fertility in Holstein Heifers. J. Dairy Sci. 85 (2002), 1633-1638

NEUMAIER, A.; GROENEVELD, E.:

Restricted maximum likelihood estimation of covariance in sparse linear models. Genet. Sel. Evol. 30 (1998), 3-26

ROTH, A.; STRANDBERG, E.; BERGLUND, B.; EMANUELSON, U.; PHILIPSSON, J.:

Genetic correlations among female fertility traits and milk production in different parties in Swedish dairy cattle. Metabolic stress in dairy cows. Occasional Publication No. 24-British Society of Animal Science, 1999

ROXSTROM, A.; STRANDBERG, E.; BERGLUND, B.; EMANUELSON, U.; PHILIPSSON, J.:

Genetic and environmental correlations among female fertility traits and milk production in different parties of Swedish red and white dairy cattle. Acta Agriculturae Scandinavica Section A-Animal Science 51 (2001) 1, 7-14

ROYAL, M.D.; PRYCE, J.E.; WOOLLIAMS, J.A.; FLINT, A.P.F.:

The Genetic Relationship between Commencement of Luteal Activity and Calving Interval, Body Condition Score, Production, and Linear Type Traits in Holstein-Friesian Dairy Cattle. J. Dairy Sci. 85 (2002), 3071-3080

SCHÖNMUTH, G.:

Realitäten in der Milchrindzüchtung. Arch. Tierz., Dummerstorf 40 (1997) Sonderheft, 48-54

VEERKAMP, R.F.; OLDENBROEK, J.K.; VAN DER GAASTAND, H.J.; VAN DER WERF, J.H.J.: Genetic Correlation Between Days Until Start of Luteal Activity and Milk Yield, Energy Balance, and Live Weights. J. Dairy Sci. 83 (2000), 577-583 
WASSMUTH, R.; BOELLING, D.; MADSEN, P.; JENSEN, J.; ANDERSEN, B.B.:

Genetic parameters of disease incidence, fertility and milk yield of first parity cows and the relation to feed intake of growing bulls. Acta Agriculturae Scandinavica Section A-Animal Science 50 (2000) 2, 93-102

Eingegangen: 10.02.2003

Akzeptiert: 03.03.2003

Anschrift der Verfasser

Prof. Dr. habil. GERHARD SEELAND, CARSTEN HENZE

Humboldt-Universität zu Berlin, Landwirtschaftlich-Gärtnerische Fakultät,

Institut für Nutztierwissenschaften, FG Züchtungsmethodik und Züchtungsplanung

Invalidenstrasse 42

D-10115 Berlin 